\title{
Heavy flavour energy loss from AdS/CFT in heavy ion collisions at RHIC and LHC
}

\author{
R Hambrock* and WA Horowitz \\ University of Cape Town, South Africa \\ E-mail: roberthambrock@gmail.com, wa.horowitzeuct.ac.za
}

\begin{abstract}
We present predictions for heavy flavour suppression, flow, and azimuthal correlations in heavy ion collisions at RHIC and LHC of two AdS/CFT based energy loss models sensitive to thermal fluctuations. While both models are in good agreement with B-meson suppression measurements from the LHC, only the model with a diffusion coefficent independent of the heavy quark's velocity is in agreement with measurements of D-meson suppression at high $p_{\mathrm{T}}$.

The partonic azimuthal correlations are compared with those from perturbative QCD based simulations [1] up to $20 \mathrm{GeV}$. When restricted to leading order production processes, we find that the strongly-coupled correlations of high transverse momentum pairs $(>4 \mathrm{GeV})$ are broadened less efficiently than the corresponding weak coupling based correlations, while low transverse momentum pairs $(1-4 \mathrm{GeV})$ are broadened with similar efficiency, but with an order of magnitude more particles ending up in this momentum class.

We thus propose heavy flavour momentum correlations as a distinguishing observable of weaklyand strongly-coupled energy loss mechanisms.
\end{abstract}

International Conference on Hard and Electromagnetic Probes of High-Energy Nuclear Collisions 30 September - 5 October 2018

Aix-Les-Bains, Savoie, France

${ }^{*}$ Speaker. 


\section{Introduction}

A key step in understanding the Quark Gluon Plasma is identifying its relevant coupling strength. Both weak and strong coupling approaches show qualitative agreement with $R_{A A}^{D}$ [2], suggesting they are attaining sufficient maturity to investigate more differential observables. We will argue that the momentum correlations of heavy quarks constitute a promising candidate as a differentiator between weakly- and strongly-coupled plasmas.

In [1], the azimuthal correlations of heavy $q \bar{q}$ pairs in a weakly-coupled plasma in $\mathrm{Pb}+\mathrm{Pb}$ collisions $\left(\sqrt{s_{N N}}=2.76 \mathrm{TeV}\right)$ were studied, both for a model involving purely collisional energy loss and one additionally incorporating radiative corrections. These weak coupling based azimuthal correlations provide a secondary indicator for the momentum correlations of heavy quarks. We will compare these correlations with two different AdS/CFT based energy loss models, one [3] with a velocity dependent diffusion coefficient, labeled $D(\mathrm{p})$, and the other [4] with a diffusion coefficient independent of the heavy quark's velocity, labeled $D_{\text {const }}$. Furthermore, we will probe the spectrum of their possible predictions with two plausible [5] 't Hooft coupling constants ( $\lambda_{1}=5.5$ and $\lambda_{2}=$ $12 \pi \alpha_{s} \approx 11.3$, with $\alpha_{s}=0.3$ ) where for the former, temperature and the Yang-Mills coupling are equated, while for the latter constant, energy density and the coupling are equated.

Finally, we will compare with measurements from LHC and provide predictions for RHIC.

\section{2. $D(\mathrm{p})$ Energy Loss Model}

The stochastic equation of motion for a heavy quark in the fluid's rest frame is [6]

$$
\frac{d p_{i}}{d t}=-\mu p_{i}+F_{i}^{L}+F_{i}^{T}
$$

where $F_{i}^{L}$ and $F_{i}^{T}$ are longitudinal and transverse momentum kicks with respect to the quark's direction of propagation and the drag loss coefficient $\mu=\pi \sqrt{\lambda} T^{2} / 2 M_{H Q}$ [7] depends on local temperature $T$, 't Hooft coupling constant $\lambda$, and heavy quark mass $M_{H Q}$. The correlations of momentum kicks are given by

$$
\begin{array}{rc}
\left\langle F_{i}^{T}\left(t_{1}\right) F_{j}^{T}\left(t_{2}\right)\right\rangle=\kappa_{T}\left(\delta i j-\frac{\vec{p}_{i} \vec{p}_{j}}{|p|^{2}}\right) g\left(t_{2}-t_{1}\right) & \left\langle F_{i}^{L}\left(t_{1}\right) F_{j}^{L}\left(t_{2}\right)\right\rangle=\kappa_{L} \frac{p_{i} p_{j}}{|p|^{2}} g\left(t_{2}-t_{1}\right) \\
\kappa_{L}=\gamma^{2} \kappa_{T}=\pi \sqrt{\lambda} T^{3} \gamma^{5 / 2} & \hat{q}=\left\langle p_{\perp}(t)^{2}\right\rangle \lambda \approx \kappa_{T} t / \lambda=\gamma\left(2 \pi T^{3} \sqrt{\lambda}\right) / v
\end{array}
$$

where $\gamma$ is the speed of the quark and g is only known numerically [3]. It should be noted that this construction does not obey the fluctuation-dissipation theorem [3].

\section{3. $D_{\text {const }}$ Energy Loss Model}

The problem with the energy loss mechanism described in 2 is that since the longitudinal momentum fluctuations grow as $\gamma^{\frac{5}{2}}$, our setup breaks down for high momenta. Via a novel calculation presented in $[4,8,9]$, we instead consider a stationary string in $A d S_{d}$ hanging into a black hole horizon and calculate $s^{2}(t, a, d)$ of the free endpoint, where $a$ parametrizes between a heavy quark for $a=0$ and a light quark for $a=1$. In $d=3$, the average squared distance travelled can be 
determined analytically for small string lengths, which is identical to the asymptotically late time behavior of a string with arbitrary initial length. We thus find the asymptotically late time behavior

$$
s^{2}(t \gg \beta, a, d)=s_{\text {small }}^{2}(t \gg \beta, a, d)=\left(\frac{d-1}{2}\right)^{2} s_{\text {small }}^{2}(t \gg \beta, a, 3)=\frac{(d-1)^{2}}{8 \pi \sqrt{\lambda}} \beta\left(1-\frac{a}{2}\right)
$$

where $\beta=T^{-1}$. At late times, the motion is diffusive, thus we can extract the diffusion coefficient

$$
D(a, d) \sim s^{2}(t \gg \beta, a, d) / 2
$$

which, in phenomenologically relevant $A d S_{5}$, for a heavy quark reads $2 \beta / \pi \sqrt{\lambda}$. This obtains

$$
\kappa_{T}=2 T^{2} / D=\pi \sqrt{\lambda} T^{2} / \beta=\pi \sqrt{\lambda} T^{3} \quad \hat{q}=\left\langle p_{\perp}(t)^{2}\right\rangle \lambda \approx \kappa_{T} t / \lambda=\left(2 \pi T^{3} \sqrt{\lambda}\right) / v
$$

Requiring these fluctuations to obey the fluctuation-dissipation theorem, we attain $\mu=\pi \sqrt{\lambda} T^{2} / 2 E$.

\section{Leading Order Azimuthal Correlations}

We compare our strong coupling azimuthal correlations to the weak coupling ones from [1] in Fig. 1. For $[10-20 \mathrm{GeV}]$, our correlations are significant more peaked at their initial backto-back correspondence. At $[4-10 \mathrm{GeV}]$, this observation still holds for the upper bound of our parameters with $\lambda_{1}=5.5$, while the $\lambda_{2}=11.3$ bounded result is of similar magnitude but looser angular correlation than either the collisional or the collisional + Bremsstrahlung based results. In the $[1-4 \mathrm{GeV}]$ range, the azimuthal correlations are almost entirely washed out for $\lambda_{2}=11.3$, while for $\lambda_{1}=5.5$, they are broadened with similiar efficiency to the weak coupling results.

Of particular interest is the difference in momentum correlations of the $[1-4 \mathrm{GeV}]$ range. At about an order of magnitude, this difference promises a distinguishing observable of weak- and strong-coupling energy loss, and should be investigated experimentally.
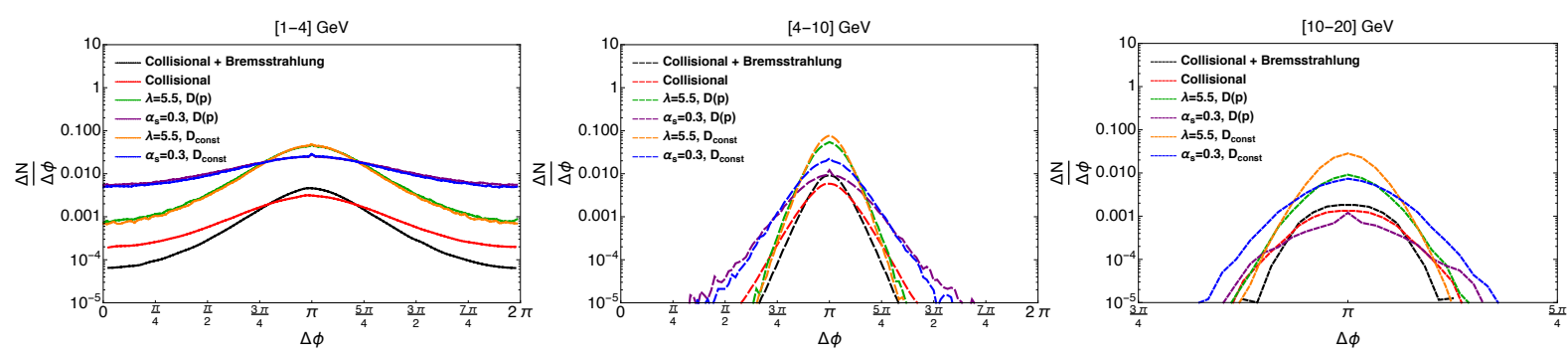

Figure 1: $\frac{d N}{d \phi}$ correlations of both pQCD- and AdS/CFT-based approaches. Of note is the order of magnitude difference in the $[1-4 \mathrm{GeV}]$ domain between the $\mathrm{pQCD}$ and AdS/CFT results.

\section{5. $R_{A A}$ and $v_{2}$}

Two crucial observables of the medium formed in heavy ion collisions are the nuclear modification factor $R_{A A}=\frac{d^{2} N_{A A} / \mathrm{d} y \mathrm{~d} p_{\mathrm{T}}}{\left\langle T_{A A}\right\rangle d \sigma_{p p} / \mathrm{dyd} p_{\mathrm{T}}}$, where $T_{A A}=\left\langle N_{p p}\right\rangle / \sigma_{p p}$ is the nuclear overlap function and 
normalizes $R_{A A}$ for trivial multiplicity scaling, and the elliptic flow $v_{2}=\frac{\int \frac{\mathrm{d}^{3} N}{\mathrm{~d}^{3} \mathbf{p}} \exp ^{i 2\left(\phi-\Phi_{R}\right)} \mathrm{d}^{3} p}{\int \frac{\mathrm{d}^{3} N}{\mathrm{~d}^{3} \mathbf{p}} d^{3} p}$, where $\Phi_{R}$ fixes the azimuthal orientation of the reaction plane.

We compare bottom and charm suppression predictions with data from CMS and ALICE (Fig. 2). While the agreement with CMS data for B meson suppression is comparable between the $D(\mathrm{p})$ and $D_{\text {const }}$ models, the comparison with ALICE data for D mesons shows the limited validity range of the $D(\mathrm{p})$ model, whereas the $D_{\text {const }}$ model remains consistent with data even for high- $p_{\mathrm{T}}$. More fundamentally, for the $D(\mathrm{p})$ model, the AdS/CFT picture naturally breaks down at $p_{\mathrm{T}} \sim 100 \mathrm{GeV}$ [3]. For the $D_{\text {const }}$ model, there is no such natural breakdown.

Fig. 3 shows our predictions for $R_{A A}$ and flow of B-mesons at RHIC. B-mesons are much less suppressed than at the LHC, due to the substantially cooler medium in heavy collisions at RHIC.
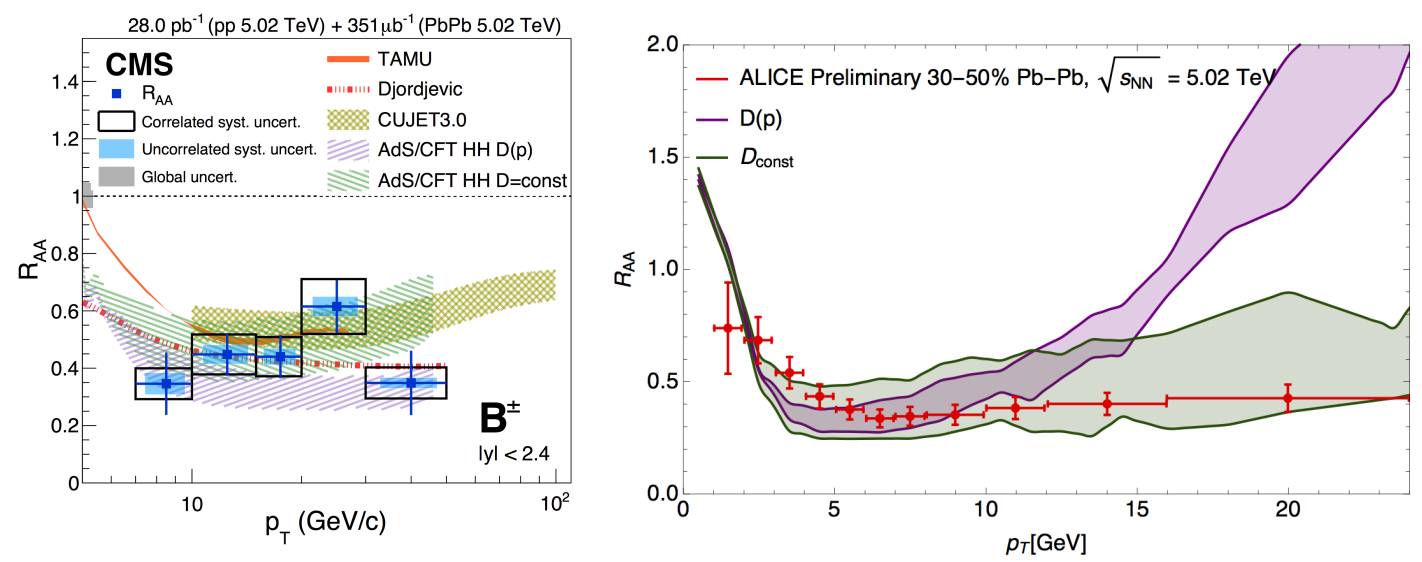

Figure 2: (Left) Comparison with $R_{A A}^{B}$ data from CMS [11] with $\sqrt{s_{N N}}=5.02 \mathrm{TeV},|\mathrm{y}|<2.4$. (Right) Comparison with $R_{A A}^{D}$ data from ALICE [12] with $\sqrt{s_{N N}}=5.02 \mathrm{TeV},|\mathrm{y}|<0.5$. The bands range from $\lambda=5.5$ to $\lambda=11.3$.
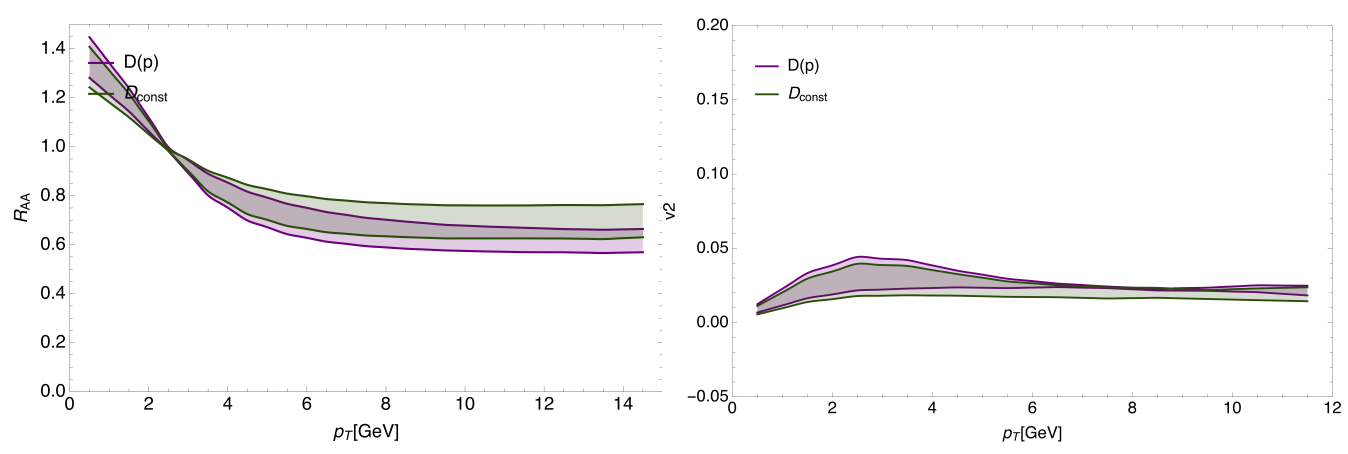

Figure 3: (Left) $R_{A A}^{B}$ and (Right) $v_{2}^{B}$ with $\sqrt{s_{N N}}=200 \mathrm{GeV},|\mathrm{y}|<1.0$ and $10-40 \%$ centrality for future RHIC measurements. The bands range from $\lambda=5.5$ to $\lambda=11.3$.

\section{Conclusion \& Outlook}

We have compared the azimuthal correlations predicted by pQCD and AdS/CFT based com- 
putations and found that, while the azimuthal correlations are qualitatively similar, their implicit momentum correlations tell a different tale. In particular, the surprise of our findings is the large dissimilarity in low momentum correlations of the pQCD and AdS/CFT based simulations; see Fig. 1 (left). Thus, bottom quark momentum correlations present an opportunity to distinguish between the energy loss mechanisms of the two frameworks.

Whether this order of magnitude difference in predictions for low $p_{\mathrm{T}}$ correlations of heavy quarks exposes weaknesses in either or both of the frameworks is inconclusive until experimental data of bottom quark momentum correlations emerge. Strong coupling approaches have fared better in the low $p_{\mathrm{T}}$ domain, where $\mathrm{pQCD}$ is restrained by uncertainties in the running coupling.

While the agreement with CMS data for B meson suppression is comparable between the $D(\mathrm{p})$ and $D_{\text {const }}$ models, the comparison with ALICE data for D mesons shows the limited validity range of the $D(\mathrm{p})$ model, whereas the $D_{\text {const }}$ model remains consistent with data even for high- $p_{\mathrm{T}}$. The RHIC data exhibits decreased suppression compared with the LHC data, which can be understood from the lower temperatures of the medium at RHIC.

The high- $p_{\mathrm{T}}$ reach of recent results from the LHC Fig. 2, particularly CMS Fig. 2 (left), exposes the limited statistics of our simulations for high- $p_{\mathrm{T}}$. In future calculations, we will migrate from aMC@NLO to POWHEG [10] to facilitate weighted event generation.

\section{Acknowledgements}

The authors wish to thank the South African National Research Foundation and SA-CERN for their generous financial support.

\section{References}

[1] Nahrgang M, Aichelin J, Gossiaux P B and Werner K 2014 Phys. Rev. C90 024907 (Preprint 1305.3823)

[2] Horowitz W A 2013 Nucl. Phys. A904-905 186c-193c (Preprint 1210. 8330)

[3] Horowitz W A 2015 Phys. Rev. D91 085019 (Preprint 1501. 04693)

[4] Moerman R W and Horowitz W A 2016 (Preprint 1605. 09285)

[5] Gubser S S 2008 Nucl. Phys. B790 175-199 (Preprint hep-th/0612143)

[6] Moore G D and Teaney D 2005 Phys. Rev. C71 064904 (Preprint hep-ph / 0412346 )

[7] Herzog C P, Karch A, Kovtun P, Kozcaz C and Yaffe L G 2006 JHEP 07013 (Preprint hep-th/0605158)

[8] Hambrock R and Horowitz W 2017 J. Phys. Conf. Ser. 889012015

[9] Hambrock R and Horowitz W A 2017 Nucl. Part. Phys. Proc. 289-290 233-236 (Preprint 1703.05845)

[10] Alioli S, Hamilton K, Nason P, Oleari C and Re E 2011 JHEP 04081 (Preprint 1012 . 3380)

[11] Sirunyan A M et al. (CMS) 2017 Phys. Rev. Lett. 119152301 (Preprint 1705. 04727 )

[12] ALICE Collaboration 2017 (Preprint ALI-PREL-128542) 